\title{
Bias in the Brain: A Diffusion Model Analysis of Prior Probability and Potential Payoff
}

\author{
Martijn J. Mulder, ${ }^{1}$ Eric-Jan Wagenmakers, ${ }^{1}$ Roger Ratcliff, ${ }^{2}$ Wouter Boekel, ${ }^{1}$ and Birte U. Forstmann ${ }^{1}$ \\ ${ }^{1}$ Cognitive Science Center Amsterdam, University of Amsterdam, 1018 TV Amsterdam, The Netherlands, and ${ }^{2}$ Department of Psychology, The Ohio State \\ University, Columbus, Ohio 43210
}

In perceptual decision-making, advance knowledge biases people toward choice alternatives that are more likely to be correct and more likely to be profitable. Accumulation-to-bound models provide two possible explanations for these effects: prior knowledge about the relative attractiveness of the alternatives at hand changes either the starting point of the decision process, or the rate of evidence accumulation. Here, we used model-based functional MRI to investigate whether these effects are similar for different types of prior knowledge, and whether there is a common neural substrate underlying bias in simple perceptual choices. We used two versions of the random-dot motion paradigm in which we manipulated bias by: (1) changing the prior likelihood of occurrence for two alternatives ("prior probability") and (2) assigning a larger reward to one of two alternatives ("potential payoff"). Human subjects performed the task inside and outside a 3T MRI scanner. For each manipulation, bias was quantified by fitting the drift diffusion model to the behavioral data. Individual measurements of bias were then used in the imaging analyses to identify regions involved in biasing choice behavior. Behavioral results showed that subjects tended to make more and faster choices toward the alternative that was most probable or had the largest payoff. This effect was primarily due to a change in the starting point of the accumulation process. Imaging results showed that, at cue level, regions of the frontoparietal network are involved in changing the starting points in both manipulations, suggesting a common mechanism underlying the biasing effects of prior knowledge.

\section{Introduction}

Perceptual decisions can be influenced by advance knowledge about the alternatives at hand. How the brain makes these biased decisions is a central issue in neuroscience (Glimcher, 2003; Lauwereyns, 2010). Formal models have been used to describe and predict bias in perceptual two-alternative forced-choice tasks (Ratcliff, 1985; Carpenter and Williams, 1995; Gold and Shadlen, 2002; Ratcliff and McKoon, 2008; Lauwereyns, 2010). Such models generally conceptualize the decision process as the accumulation of sensory information over time toward a decision threshold (Fig. 1A; for review, see Bogacz, 2007; Gold and Shadlen, 2007; Ratcliff and McKoon, 2008; Wagenmakers, 2009). Two possible mechanisms for bias have been proposed: (1) prior knowledge leads to shifts in the starting point of the evidence accumulation process (Edwards, 1965; Laming, 1968; Link and Heath, 1975; Ratcliff, 1985; Voss et al., 2004; Bogacz et al., 2006; Diederich and Busemeyer, 2006; Wagenmakers et al., 2008b) or (2) prior knowledge affects the rate with which sensory evidence is accumulated

Received Aug. 11, 2011; revised Dec. 15, 2011; accepted Dec. 26, 2011.

Author contributions: M.J.M., E.-J.W., and B.U.F. designed research; M.J.M. and W.B. performed research; M.J.M., E.-J.W., and R.R. analyzed data; M.J.M. wrote the paper.

This research was supported by VENI and VIDI grants from the Netherlands Organization for Scientific Research (B.U.F. and E.J.W., respectively). We thank Leendert van Maanen, Gilles Dutilh, and Joachim Vandekerckhove for methodological discussion, and we thank Steven Scholte and Diederick Stoffers for helpful tips on using FSL.

The authors declare no competing financial interests.

Correspondence should be addressed to Dr. Martijn Mulder, Cognitive Science Center Amsterdam, University of Amsterdam, Plantage Muidergracht 24, 1018 TV Amsterdam. E-mail: m.j.mulder@uva.nl.

DOI:10.1523/JNEUROSCI.4156-11.2012

Copyright $\odot 2012$ the authors $\quad 0270-6474 / 12 / 322335-09 \$ 15.00 / 0$ over time (Ashby, 1983; Ratcliff, 1985; Diederich and Busemeyer, 2006; Fig. $2 B$ ). Both scenarios result in faster and more choices toward the alternative that is a priori more likely or more profitable, and previous studies have suggested that knowledge about a choice alternative's probability of occurrence or potential payoff can invoke either scenario (Gold and Shadlen, 2002; Simen et al., 2006). Furthermore, for both types of prior knowledge, imaging studies have shown that regions of the frontoparietal and frontostriatal circuits play a role in biasing choice behavior (Boettiger et al., 2007; Serences, 2008; Summerfield and Koechlin, 2008; Basten et al., 2010; Fleming et al., 2010a,b; Forstmann et al., 2010; Philiastides et al., 2010; Preuschhof et al., 2010; Scheibe et al., 2010; Summerfield and Koechlin, 2010). However, only a small subset of these studies quantified bias by using a model-based approach (Basten et al., 2010; Forstmann et al., 2010; Summerfield and Koechlin, 2010). Furthermore, to our knowledge, no studies used manipulations of both prior probability and potential payoff to test there is a common neural substrate underlying these different types of bias in perceptual decision making.

In this study, we first investigated whether the two types of prior knowledge have a similar effect on either the starting point or the rate of evidence accumulation of the decision process. Second, we studied whether there is a common neural substrate that gives rise to changes in these parameters. We used two versions of the random-dot motion paradigm where we induced bias by (1) changing the likelihood that one of the alternatives is correct (prior probability) and (2) assigning a larger reward to one of the alternatives (potential payoff; Fig. 3). Bias was quantified by fitting the drift-diffusion model 
(DDM) to response time (RT) and accuracy data. We then used individual differences in the model parameters to identify a common network of brain regions involved in biasing perceptual decisions.

\section{Materials and Methods}

Subjects. Twenty-three healthy subjects (14 female, mean age $=23.8, \mathrm{SD}=3.3)$ performed a random-dots motion paradigm in a $3 \mathrm{~T}$ scanner. In addition, subjects also performed an extra behavioral session outside the scanner environment. Subjects were recruited through the University of Amsterdam and had normal or corrected-to-normal vision. The procedure was approved by the ethical review board at the University of Amsterdam and informed consent was obtained from each subject. Three subjects were excluded due to scanner artifacts or because their performance was at chance level. A total of 12 female and 8 male subjects (mean age $=23.7, \mathrm{SD}=3.0$ ) were included in the final analyses. Eighteen subjects were right-

handed and 2 subjects were left-handed as confirmed by the Edinburgh Inventory (Oldfield, 1971). According to self-report, no subject had a history of neurological, major medical, or psychiatric disorder.

Stimuli. Subjects performed an RT version of a random-dot motion direction-discrimination task. Bias was manipulated by a cue indicating a higher probability of the direction of the motion stimulus or a higher value associated with the motion stimulus (Fig. 3). Subjects were instructed to maintain fixation on a cross on the middle of the screen, pay attention to the cue, and decide the direction of motion of a cloud of randomly moving white dots on a black background. They were to indicate their decision at any time during motion viewing with a left or right button press. The motion stimuli were similar to those used previously (Newsome and Paré, 1988; Britten et al., 1992; Gold and Shadlen, 2003; Palmer et al., 2005; Ratcliff and McKoon, 2008; Mulder et al., 2010): white dots, with a size of $3 \times 3$ pixels, moved within a circle with diameter of $5^{\circ}$ with a speed of $5^{\circ} / \mathrm{s}$ and a density of $16.7 \mathrm{dots} / \mathrm{deg}^{2} / \mathrm{s}$ on a black background. On the first three frames of the motion stimulus, the dots were located in random positions. For each of these frames the dots were repositioned after two subsequent frames (the dots in frame 1 were repositioned in frame 4 , the dots in frame 2 were repositioned in frame 5, etc.). For each dot, the new location was either random or in line with the motion direction. The probability that a dot moved coherent with the motion direction is defined as coherence. For example, at a coherence of $50 \%$, each dot had a probability of $50 \%$ to participate in the motionstimulus, every third frame (see also Britten et al., 1992; Gold and Shadlen, 2003; Palmer et al., 2005).

Visual stimuli were generated on a personal computer (Intel Core2 Quad 2.66 GHz processor, 3GB RAM, two graphical cards: NVIDIA GeForce 8400 GS and a NVIDIA GeForce 9500 GT, running MS Windows XP SP3) using custom software and the Psychophysics Toolbox Version 3.0.8 (Brainard, 1997; Pelli, 1997) for Matlab (version 2007b, MathWorks). For the session outside the scanner environment, the dots were presented on a 51.3-cm-wide LCD screen at a viewing distance of 50 $\mathrm{cm}$. In the scanner session, the dots were presented on a $61-\mathrm{cm}$-wide projection screen using a projector with a resolution of $1920 \times 1200$ (GeForce 9500 GT graphical card) and adjusted to match the same diameter $\left(5^{\circ}\right)$ as the session outside the scanner.

To match the difficulty level of the motion stimulus across subjects, each subject performed a block of 200 trials of randomly interleaved stimuli with different motion strengths (respectively, $0,10,20,40$, or $80 \%$ coherence, 40 trials each). We fitted the proportional-rate diffusion model to the mean response times and accuracy data of this block using a maximum likelihood procedure (Palmer et al., 2005). For each subject, the motion strength at $80 \%$ accuracy was then interpolated from the

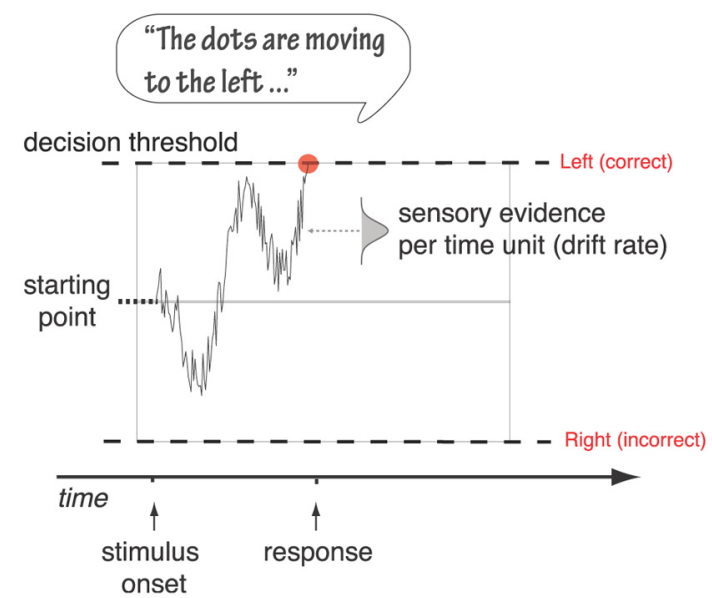
psychometric curve (predicted by the proportional-rate diffusion model;
Palmer et al., 2005) and used in the remaining experimental blocks. This procedure was done before the experimental blocks for both the inside and outside scanner session. Subjects did not differ in the $80 \%$ performance levels or in the main parameters of the proportional-rate diffusion model (decision threshold, sensitivity, or non-decision time; Palmer et al., 2005) between the inside and outside scanner sessions (paired samples $t$ tests: $t_{(19)}<1.72, p>0.1$ ).

Manipulations of bias by prior probability and potential payoff. Prior information was given in the form of a cue. The cue was either an arrow pointing to the left or to the right, or a square with the size of the arrow without arrow-points for the unbiased condition. For the probability manipulation, a percentage sign "\%" was printed at the middle of the cue (Fig. 3). For the payoff manipulation this was a euro sign " $€$." One block consisted of 40 bias trials and 40 neutral trials (with 20 leftward and 20 rightward directed trials each). In the prior probability blocks, 32 (16 leftward cues and 16 rightward cues) of 40 bias trials were valid i.e., the direction indicated by the cue was consistent with the direction of the stimulus ( $80 \%$ valid), and 8 ( 4 leftward cues and 4 rightward cues) of 40 bias trials where invalid, i.e., cue and stimulus direction were inconsistent (20\% invalid). Subjects received 5 points for each correct response in both biased and neutral trials. No points were given for an incorrect response.

In the potential payoff blocks, 20 (10 leftward cues and 10 rightward cues) of 40 bias trials were valid, i.e., cue and stimulus direction were consistent, and a large reward was received for a correct response (8 points, valid). In 20 (10 leftward cues and 10 rightward cues) of 40 trials, the cue and stimulus direction were inconsistent and subjects received 2 points for a correct response ( 2 points, invalid). No points were given for an incorrect response. Eight extra "null" trials were randomly added to each block in the scanner-session to improve estimation of the evoked response in the MR signal. In these null trials, blank cues (no prior information) were followed by a stimulus and feedback was given by either printing the word "correct" or "incorrect" on the screen (no points). Subjects were paid a fixed amount for both the behavior and scanner sessions. Before the experiment, subjects were told that they could earn up to 10 extra euros if performance was perfect.

Paradigm timing. In the scanner-session, subjects performed 2 blocks of each version of the random-dots motion task. Each trial started at the beginning of a volume acquisition. Trial timing was optimized for fMRI analyses. A fixation cross was presented with a randomly chosen duration of either 100, 350, 800, or $1200 \mathrm{~ms}$. Next, the cue was presented for 1000 ms followed by a fixation cross with a randomly chosen duration of 3400 , 4000,4500 , or $5000 \mathrm{~ms}$. The motion stimulus was then presented during 


\section{A Biasing effects in the decision process}

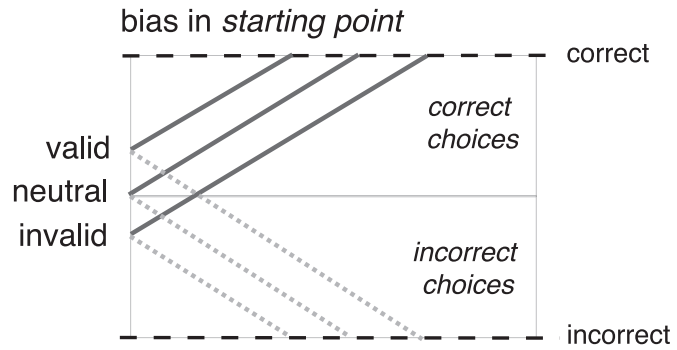

\section{B Expected effects}
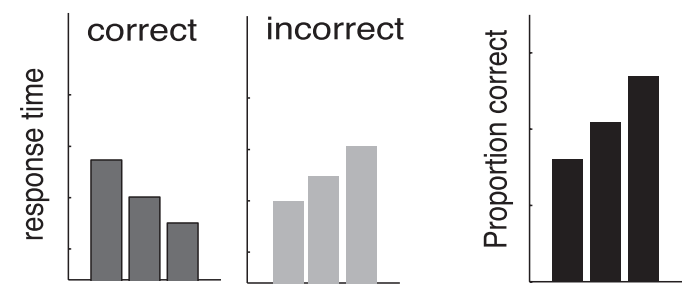

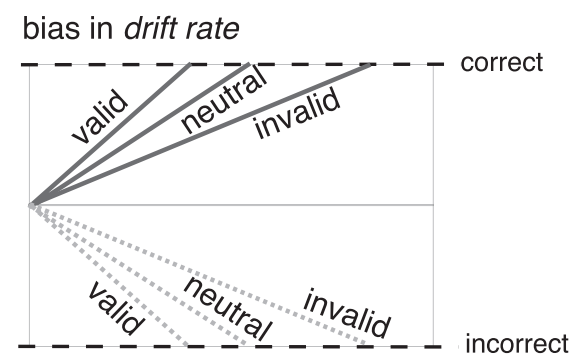

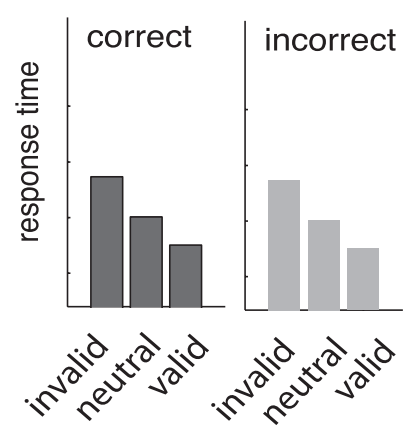

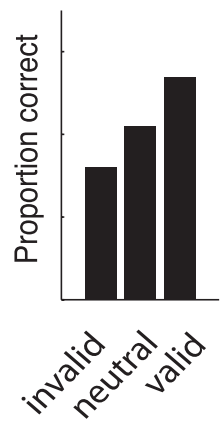

Figure 2. Possible effects of bias on choice behavior. $A$, Effects of bias explained by the drift-diffusion model. When prior information is valid for the choice at hand, subjects will have faster and more correct choices, whereas invalid information results in slower and less correct choices compared with choices where no information is provided (neutral). These effects can be explained by changes in the starting point or the drift rate of the accumulation process. $\boldsymbol{B}$, Expected effects of bias on RT and accuracy data for choices with valid, neutral and invalid cues. Note that bias effects for each of these parameters will result in a different pattern of RTs for incorrect choices. For example, when a valid cue shifts the starting point toward the correct bound, there is a greater distance for the accumulation process to hit the incorrect bound. In contrast, when the drift rate is biased by a valid cue, the rate toward the incorrect bound is increased, resulting in faster RTs for incorrect choices.

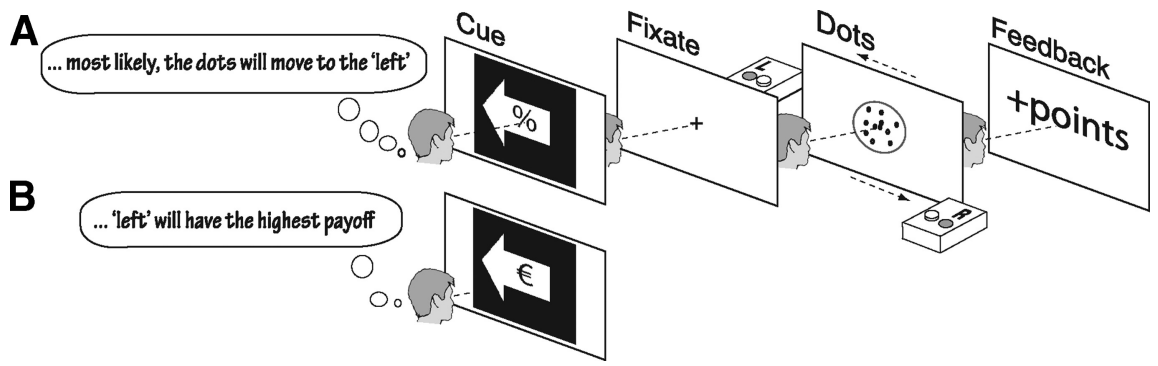

Figure 3. Two versions of the random-dots motion task where choice bias was manipulated by providing information about the likelihood of the direction of the stimulus (prior probability) $(\boldsymbol{A})$ or the value associated with the direction of the stimulus (potential payoff) (B).

which subjects could choose the direction by pressing the left or right button. The stimulus remained on the screen for a total duration of 1500 $\mathrm{ms}$. Following the stimulus, feedback was presented for $500 \mathrm{~ms}$, showing the number of points earned by the subject. Responses faster than $100 \mathrm{~ms}$ were considered premature and followed by the words "too fast" on the screen. When subjects failed to respond within the $1500 \mathrm{~ms}$ stimulus duration they received a feedback showing the word "miss." This was followed by the fixation cross during a short filler time to fix the total trial time to 8 or $10 \mathrm{~s}$. For the session outside the scanner environment jitter times were fixed at $100 \mathrm{~ms}$. Here, subjects performed 4 blocks of each version of the task. For both the inside and outside scanner sessions, block-order was interleaved and counterbalanced across subjects.

Behavioral analyses. Descriptive results of the behavioral data were obtained using PASWStatistics (version 18.0, SPSS Inc.). For each manipulation (prior probability and potential payoff), trials with left and rightward stimuli were collapsed and classified as correct and incorrect valid, invalid and neutral trials. The mean accuracy and median RT were then computed for each subject separately. Moreover, mean accuracy and median RTs were computed separately for sessions inside and outside the scanner, resulting in 4 conditions (inside and outside scanner $\times$ prior and payoff manipulation). To investigate whether both manipulations had similar effects on performance, we tested for overall differences between the two manipulations using a $2 \times 3$ repeated-measures ANOVA with condition (prior and payoff) and trial-type as factors. Since we expected a specific linear pattern for RTs and accuracy across trial-type (valid, neutral, and invalid trials; Fig. 2), a $1 \times 3$ repeated-measures ANOVA for each condition separately was performed.

Fitting the drift diffusion model to the data. The DDM assumes that for two-alternative forced choice decisions, sensory evidence in favor of one of the alternatives begins to accumulate from a starting point $z$. When the evidence accumulation process (quantified by drift rate $v$ ) reaches a threshold value $(a)$, a response is initiated. The full DDM consists of seven parameters: three parameters for the decision process (decision threshold $a$, mean starting point $z$; henceforth: "starting point," and mean drift rate $v$; henceforth: "drift rate," a parameter for non-decision processes (non-decision time Ter), and three parameters for across-trial variability (variability in starting point $s z$, variability in non-decision time $s t$, and variability in stimulus quality $\eta$; Ratcliff, 1978; Ratcliff and Tuerlinckx, 2002; Ratcliff and McKoon, 2008). Additionally, one param- 
eter was used to account for "contaminant" responses-slow outlier response times that do not come from the decision process of interest (e.g., responses influenced by lack of attention; Ratcliff and Tuerlinckx, 2002).

The DDM can capture the effects of bias either by changes in starting point $(\Delta z)$ or by changes in $\operatorname{drift}$ rate $(\Delta v$; Fig. 2 ; see Fig. $5 A)$. Hence, the starting-point and drift bias-parameters $(\Delta z, \Delta v)$ were allowed to vary across valid, neutral, and invalid choices with the restriction that for neutral trials $\Delta z$ and $\Delta v$ equaled zero. In addition, for neutral trials we assumed that starting point $z$ equals half the decision threshold $a$. For starting point we assume that it is biased by the cue toward the correct bound $(z+\Delta z)$ on valid trials, and away from the correct bound $(z-\Delta z)$ on invalid trials. Similarly, for drift rate we assumed that it is biased by the cue toward the correct bound $(v+\Delta v)$ on valid trials and away from the correct bound $(v-\Delta v)$ on invalid trials (see Fig. $5 A$ ).

We fitted the DDM to the data from each subject and each condition separately (prior probability and potential payoff, both inside and outside the scanner environment). First, for each trial-type (valid, neutral and invalid) RT data were divided by RT-bins defined by the 10th, 30th, 50th, 70th, and 90th quantiles of the RT-distribution for correct and incorrect responses. RT-bins together with the proportion correct responses for each bin were entered in a Fortran routine that minimizes a $\chi^{2}$ value using a Nelder-Mead SIMPLEX optimization algorithm (Ratcliff and Tuerlinckx, 2002). Bias was quantified as the proportional change in the mean starting-point $(\Delta z / z)$ and the mean drift rate $(\Delta v / v)$. Nonparametric tests were used to assess whether the medians of bias were significantly different from zero.

Imaging acquisition. Imaging data were acquired on a 3T Philips scanner using a 32-channel head coil. For each subject, a T1 anatomical scan was acquired [T1 turbo field echo, 220 coronal slices of 1 $\mathrm{mm}$, with a resolution of $1 \times 1 \mathrm{~mm}$, field of field of view $=240 \times$ $188 \times 220 \mathrm{~mm}$, flip angle $\left.=8^{\circ}, \mathrm{TR}=8.4 \mathrm{~ms}, \mathrm{TE}=3.9 \mathrm{~ms}\right]$. For functional imaging, 2D EPI scans were acquired in sagittal orientation with forty-one $3 \mathrm{~mm}$ slices with in-plane resolution of $3 \times 3 \mathrm{~mm}$ [field of view $=192 \times 64 \times 64 \mathrm{~mm}, \mathrm{TR}=2000 \mathrm{~ms}, \mathrm{TE}=27.63 \mathrm{~ms}$, flip-angle $=90^{\circ}$, voxel size $=3 \times 3 \times 3 \mathrm{~mm}$ ].

fMRI analyses. fMRI analyses were performed using FEAT (FMRI Expert Analysis Tool) Version 5.98, part of FSL (version 4.1, FMRIB's Software Library, www.fmrib.ox.ac.uk/fsl). The first three volumes were discarded due to possible scanner-drift effects. The remaining images were then realigned to compensate for small head movements (Jenkinson et al., 2002). Data were spatially smoothed using a $5 \mathrm{~mm}$ FWHM Gaussian kernel. The data were temporally filtered using a high-pass filter with a cutoff frequency of $1 / 100 \mathrm{~Hz}$ to correct for baseline drifts in the signal. Finally, the functional data were prewhitened using FSL (FMRIB's Software Library, www.fmrib.ox.ac.uk/ fsl; Woolrich et al., 2001). All functional datasets were individually registered into $3 \mathrm{D}$ space using the subjects' individual high-resolution anatomical images acquired at the beginning of each scanning session. The individual 3D reference dataset was used to normalize the functional data into MNI space by linear scaling (affine transformations; Jenkinson and Smith, 2001). The statistical evaluation was performed using the general linear model. The design matrix was convolved using a double gamma hemodynamic response function and its temporal derivative.

Note that at the cue level there is no distinction between valid and invalid trials: only when a stimulus is presented can a trial be identified as valid or invalid. As such, for the cue regressors, we collapsed the valid and invalid trials in a single bias regressor assuming that the information provided by the cue will bias the system independent of the following stimulus. Accordingly, stimuli were divided into valid and invalid regressors. Furthermore, cues were divided by direction (left or right) to test for possible effects of the direction of the motion stimulus. That is, in addition to a sufficient duration of the jitter times between cue and stimuli onsets (see Paradigm timing), we controlled for a possible effect of stimulus direction at cue level by testing for differences between left and right neutral cues. Since no direction was provided in these cues, a possible directional stimulus effect would result in significant signal chance for left versus right (or vice versa) contrast images. No such effect was found, suggesting that jitter times were sufficient to separate cue from stimulusrelated BOLD responses.
In sum, first level analyses were conducted for individual blocks of each subject using a GLM with 13 regressors: 5 cue regressors (left and right for bias and neutral cues, and one extra regressor for null cues), 5 stimulus regressors (valid, invalid, neutral, incorrect/miss, and null trials), and 3 feedback regressors (correct, incorrect $/ \mathrm{miss}$, and null).

For each subject we created bias versus neutral cue contrast images that were entered in the higher-level analyses. Higher-level analyses were performed using FLAME1 + 2 (FMRIB's Local Analysis of Mixed Effects; Beckmann et al., 2003; Woolrich et al., 2004). To test for specific changes in BOLD response due to individual differences in bias, we used the bias terms that resulted from the DDM analyses as covariates in the fMRI analyses. A vector of the proportional change in starting point $(\Delta z / z)$ for all subjects was de-meaned and entered in covariate analyses in the GLM for each manipulation separately. Bias versus neutral cue contrast images were entered as the dependent variable. Additionally, we added the individual proportional changes in drift rate $(\Delta v / v)$ as a nuisance regressor. Results are reported at a cluster corrected threshold of $z>2.6(p<0.05$, using Gaussian random field theory), or at a more lenient uncorrected threshold of $z>2.6$, with a cluster extent of $k>20$ for the exploratory analyses. To investigate whether there are common bias-sensitive regions for both conditions, we ran a conjunction analyses across the statistical images of the above described covariate analyses (Nichols et al., 2005). Results are reported at an uncorrected threshold of $z>2.3$, with a cluster extent of $k>5$.

\section{Results}

We investigated whether two types of prior knowledge have a similar effect on either the starting point or the rate of evidence accumulation of the decision process. We further investigated whether there is a common neural substrate that gives rise to these effects. Two versions of the random-dot motion paradigm were used. In these versions we manipulated bias by changing the prior probability or the potential payoff for one of the alternatives. Subjects performed the task inside and outside a 3T MRI scanner.

Based on the dynamics of the decision process accounted by the DDM, we expect specific effects for RT and accuracy data when choices are biased (Fig. 2). Furthermore, the pattern of RTs for incorrect choices across trials with invalid, neutral, and valid cues will be qualitatively different for a starting-point or a driftrate effect (Fig. $2 \mathrm{~B}$ ). Below, we first report the descriptive behavioral results for both bias manipulations inside and outside the scanner environment. Next, we report quantitative measures of bias obtained from the DDM. Finally, we report results of the model-based $\mathrm{fMRI}$ analyses where we used the quantitative biaseffects to identify brain regions that are sensitive to cue-induced bias.

\section{Descriptive results \\ Effects of bias}

To investigate the effects of bias-manipulation we entered the individual accuracy (proportion correct) and median RTs for correct and incorrect choices in a $1 \times 3$ repeated-measures ANOVA for each manipulation (prior probability and potential payoff) and each session (outside and inside the scanner environment) separately. Results are shown in Figure 4.

\section{Outside scanner}

For the session outside the scanner, there was a main effect of bias on accuracy $\left(F_{(1,19)}>5.01, p<0.05\right)$ and RTs for correct choices $\left(F_{(1,19)}>10.23, p<0.001\right)$ for both the prior probability and potential payoff manipulations. No significant effects were found on RTs for incorrect choices $\left(F_{(1,19)}<1.21\right.$, $p>0.31$ ). Linear trends across trail-type (invalid, neutral, valid) were significant for RTs for correct choices and accuracy 

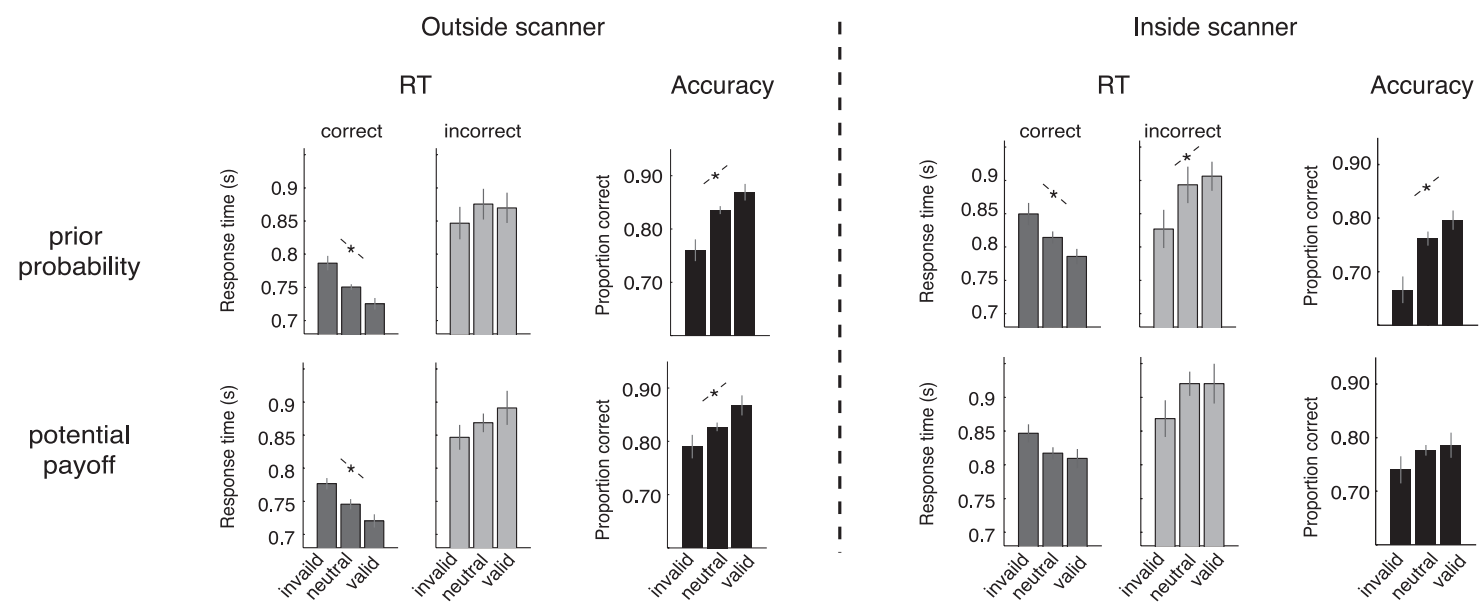

Figure 4. Effects of bias induced by prior probability and potential payoff in accuracy and RTs for correct and incorrect choices. Asterisks indicate a significant linear trend across trial-types. Error bars represent $1 \mathrm{SE}$ from the mean.

(respectively, $F_{(1,19)}>15.38, p=0.001$ and $F_{(1,19)}>5.62, p<$ $0.05)$ but not for RTs for incorrect choices $\left(F_{(1,19)}<1.61, p>\right.$ $0.22)$. No difference was found for RT and accuracy between the prior probability and potential payoff manipulation (all $F$ values $<1.39$, all $p$ values $>0.26$ ).

\section{Inside scanner}

For the session inside the scanner, we found a significant effect of bias for the prior probability manipulation on accuracy and correct RTs $\left(F_{(1,19)}>6.17, p=0.005\right)$. Linear trends across trial-type were significant for accuracy, correct and incorrect RTs $\left(F_{(1,19)}>\right.$ $5.12, p<0.05)$. For the manipulation with potential payoff, the effect of bias and the linear trend across trial-type were at trend level for correct RTs $\left(F_{(1,19)}>2.61, p=0.09\right)$. No significant bias effect was found on accuracy and incorrect RTs $\left(F_{(1,19)}<1.39\right.$, $p>0.26)$. We also did not find a difference for RT between the prior probability and potential payoff manipulation $\left(F_{(1,19)}=\right.$ $0.41, p=0.53)$. For accuracy, the difference between the two manipulations reached trend level $\left(F_{(1,19)}=3.245, p=0.088\right)$ with overall more correct choices for the potential payoff manipulation.

In sum, descriptive results show that for most conditions, subjects were biased toward the most likely and most rewarding alternative resulting in more correct and faster choices for trials where the cue was valid compared with invalid cues. Furthermore, the pattern of RTs for incorrect choices in all four conditions suggests that these bias effects can be explained best by a change in starting-point of the evidence accumulation, as shown in Figure 2. To further quantify this effect we fitted the driftdiffusion model to the RT and accuracy data.

\section{Quantification of bias using the DDM}

To quantify the effects of bias in the dynamics of the decision process, we fitted the DDM to the data for each condition separately (prior probability and potential payoff, inside and outside the scanner environment). Both bias parameters $\Delta z$ for startingpoint and $\Delta v$ for drift rate were allowed to vary across the different trial-types (see Materials and Methods; Fig. 5A). Bias was calculated as the proportional change in starting point $(\Delta z / z)$ and the proportional change in drift rate $(\Delta v / v)$. For both bias manipulations we found a significant increase in starting point across subjects in the session outside the scanner (median $\left[25^{\text {th }}\right.$ $75^{\text {th }}$ quartiles $]$ value of $(\Delta z / z)$ for prior probability $=0.07[-0.01$ 0.213 , and for potential payoff $=0.153$ [0.040 0.280], Wilcoxon
Signed Rank test for $\mathrm{H} 0$ : median value of proportional bias $=0$, $p<0.05)$. For the scanner session we found significant effects for both manipulations as well (median $\left[25^{\text {th }} 75^{\text {th }}\right.$ quartiles] value of $(\Delta z / z)$ for prior probability $=0.178[0.8800 .318]$, and for potential payoff $=0.117[0.0050 .305], p<0.01)$. No significant effects were found for the proportional change in drift rate. These results suggest that in this experiment, choice bias is mainly driven by adjusting the starting point of the accumulation process rather than changing the rate of accumulation itself (Fig. $5 B$ ).

\section{Imaging results: a diffusion model analyses of bias}

To identify regions that are involved in the neural mechanism underlying bias in the decision process, we added a covariate with the proportional increase in starting point for each subject to the GLM of the whole-brain analyses (see Materials and Methods). For the prior probability manipulation, we found BOLD responses that were related to changes in starting point in the right superior frontal gyrus (SFG), right middle frontal gyrus (MFG), left inferior frontal gyrus (IFG) and left intraparietal sulcus (IPS): subjects with a larger proportional increase in starting point showed higher BOLD signal in these frontoparietal regions. Furthermore, medial frontal gyrus (medFG) and anterior cingulate gyrus (ACG) were more active for subjects with larger starting point effects as well (Fig. 6)

For the bias manipulation with potential payoff, no regions were found that survived the cluster corrected threshold $(z=2.6$, $p=0.05$ ). However, as behavioral studies of tasks with similar bias manipulations have shown weaker effects for the payoff than for the prior manipulation (Maddox and Bohil, 1998; Simen et al., 2009; Leite and Ratcliff, 2011) we applied a more lenient threshold (uncorrected $z>2.6, k>20$ ). According to this more exploratory analysis the effect of variability in starting point was evident in similar regions as found in the prior probability manipulation. In addition to regions of the frontoparietal circuit, for the payoff manipulation we also found increased BOLD responses in orbitofrontal cortex (OFC), the hippocampus and occipital lobe (Fig. 7).

As both prior probability and potential payoff showed similar brain regions that are sensitive to changes in starting point, we performed a conjunction analyses across both bias manipulations to identify regions that are sensitive to changes in starting point, regardless of the type of prior information involved. Results show regions of the frontoparietal circuit that were sensitive 
to starting-point changes for both manipulations, suggesting a common network involved in the underlying mechanism of bias in choice behavior (Fig. 8).

\section{Discussion}

We used model-based functional MRI to investigate whether bias in choice behavior is similar for different types of prior knowledge. We used two versions of the random-dots motion paradigm where bias was manipulated by either (1) changing the prior likelihood of occurrence for two alternatives (prior probability), and (2) assigning a larger reward to one of two alternatives (potential payoff). Subjects performed the task in two sessions with the first session being optimized to measure brain activity using functional MRI. Bias was quantified by fitting the DDM to the RT and accuracy data for each manipulation and session separately. These measures were then used to identify brain regions that are sensitive to bias-induced changes in the dynamics of the decision process.

For most sessions we found significant linear trends for RT and accuracy across trials with valid, neutral, and invalid cues: subjects made faster and more correct choices for trials with valid compared with invalid cues. For the potential payoff manipulation inside the scanner the linear trend did not exceed the level of significance. Nevertheless, fitting the DDM to the data showed a similar proportional increase in starting point for both manipu-

lations inside and outside the scanner environment, suggesting that the bias manipulation was effective for both prior probability and potential payoff conditions.

When comparing RT patterns for correct and incorrect choices, the effects for incorrect choices on RT were opposite compared with correct choices: in most but not all conditions, subjects made slower incorrect choices for valid trials and faster incorrect choices for invalid trials. This effect indicates that bias in these manipulations is incorporated in the decision process by a change of the starting point of the accumulation process (Fig. 2 ). When the starting point shifts toward the bound of the alternative that is represented by the cue, the distance to the other alternative is larger resulting in slower RTs for trials with invalid cues. Fitting the DDM to the data confirmed this effect: for both manipulations the starting point effects were significant in each session whereas no such effect was found for the effects of bias on drift rate. Together, these results suggest a common mechanism underlying bias by prior knowledge in choice behavior. Findings are in line with earlier studies that used model-based approaches to investigate bias in choice behavior (Laming, 1968; Link and Heath, 1975; Ratcliff, 1985; Ratcliff et al., 1999; Voss et al., 2004; Palmer et al., 2005; Bogacz et al., 2006; Wagenmakers et al., 2008a; Forstmann et al., 2010; Summerfield and Koechlin, 2010).

Next, we identified brain regions that are susceptible to the model-based bias mechanism. We informed the fMRI analyses with quantitative measurements of bias in each manipulation.
B proportional increase in parameters
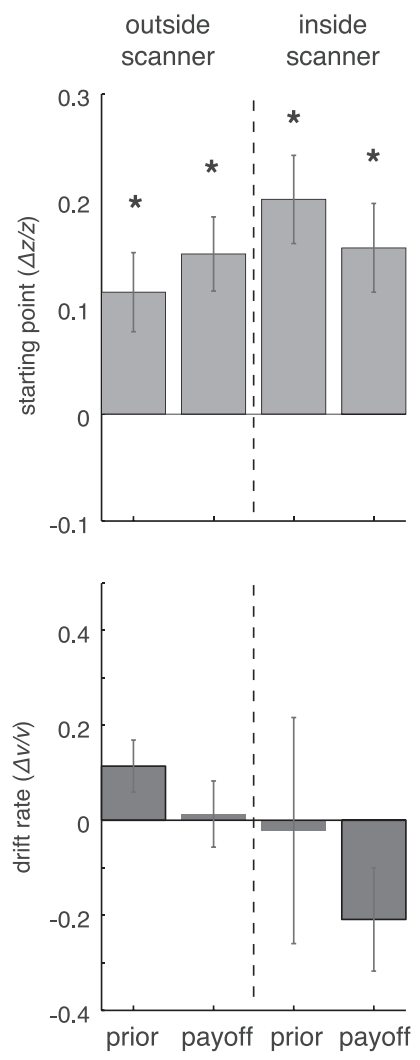

Bias effects $(\Delta v)$ in drift rate

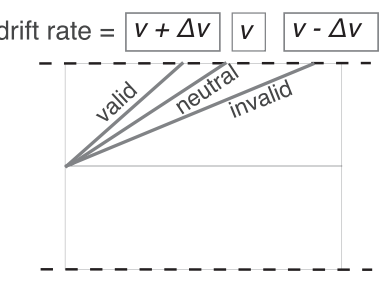
ias effect $(\Delta z)$ in starting point

Figure 5. Effects of bias in DDM parameters. $\boldsymbol{A}$, Bias is measured in starting point $z$, which is assumed to be half-way threshold (bottom) for prior probability and potential payoff, in and outside the scanner environment. Results show significant effects for changes in the starting point, but not for changes in the drift rate. Error bars represent $1 \mathrm{SE}$ from the mean.

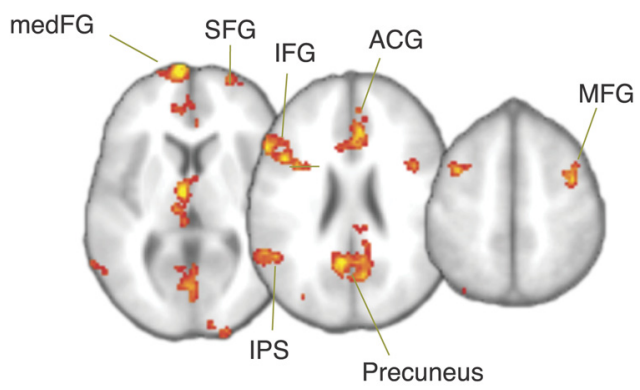

Figure 6. BOLD responses for starting point changes in the prior probability manipulation (cluster corrected, $z>2.6, p<0.05$ ).

This allowed us to study the specific bias effects of the prior information reflected in the cue, rather than a general effect of the cue itself (Forstmann et al., 2011). For the prior probability manipulation we found regions in the frontoparietal network that were sensitive to changes in starting point. These results are in line with those from earlier studies that investigated bias in choice behavior: regions such as SFG, MFG, left IFG and IPS have been associated with decision processes (Heekeren et al., 2004, 2006; Philiastides and Sajda, 2007; Ho et al., 2009; Kayser et al., 2010) and prior or value-induced bias in decision making (Boettiger et al., 2007; Serences, 2008; Basten et al., 2010; Preuschhof et al., 


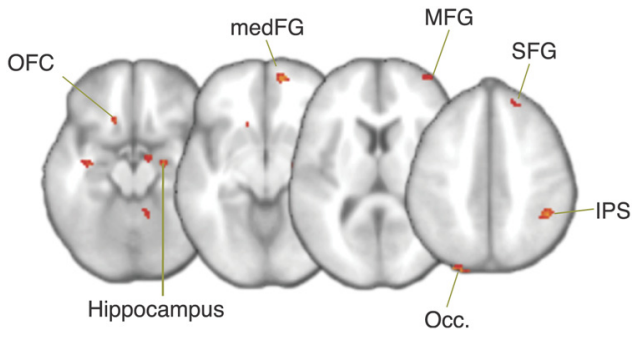

Figure 7. BOLD responses for starting point changes in the potential payoff manipulation (uncorrected, $z>2.6$, with cluster extent $>20$ ). Occ., Occipital lobe.

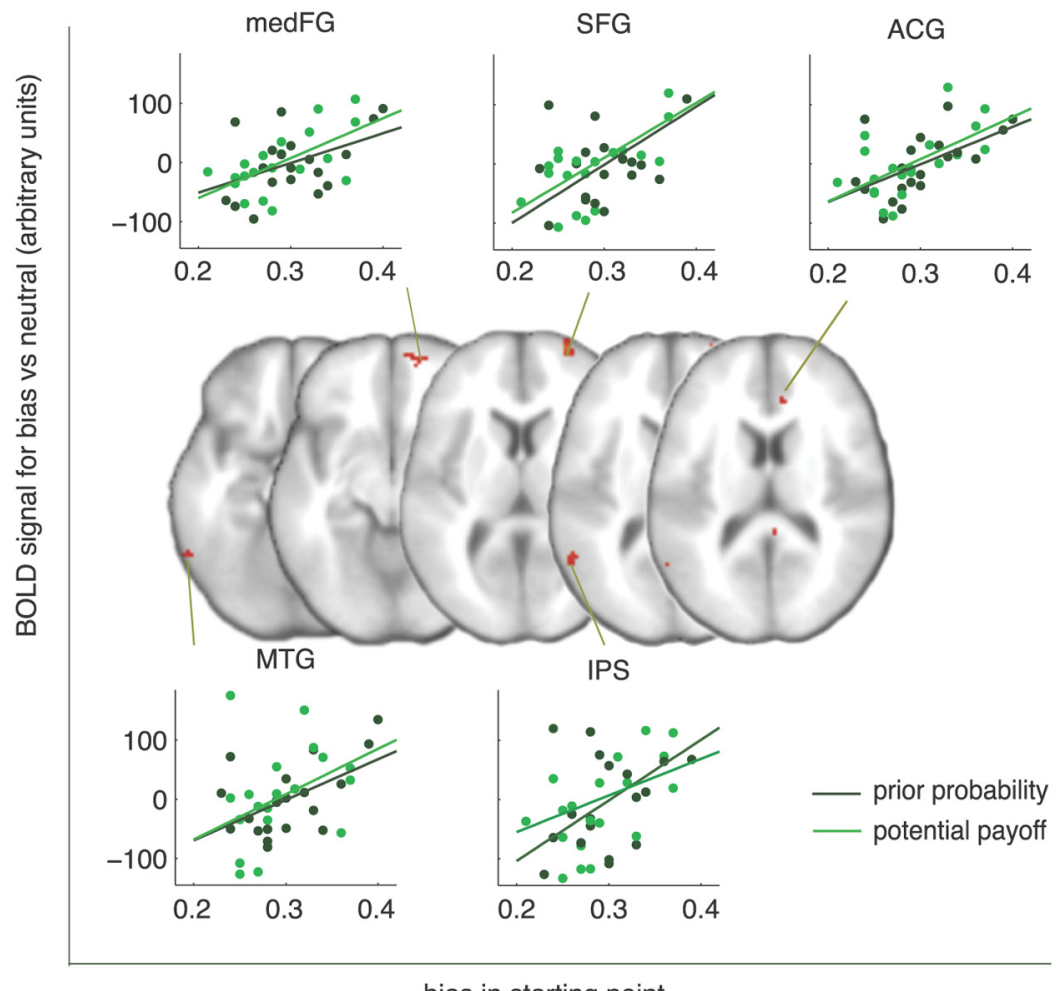

bias in starting point

Figure 8. BOLD responses for regions that were sensitive to starting point changes in both bias manipulations (conjunction, uncorrected, $z>2.3$, with cluster extent $>5$ voxels). For visualization purposes we added a graphical representation of the relationship between changes in starting point and changes in BOLD signal for the prior probability and potential payoff manipulation. Data points are derived from individual subjects. These results suggest a common network involved in the underlying mechanism of bias in choice behavior. MTG, Middle temporal gyrus.

2010; Scheibe et al., 2010; Summerfield and Koechlin, 2010; Hansen et al., 2011). Furthermore, we found increased BOLD responses in occipital regions which have been associated with value-based decision making (Serences, 2008; Summerfield and Koechlin, 2008).

Other regions, such as the medFG and ACG, were sensitive to starting point changes as well. The medFG and ACG regions have been associated with processing prior information in decision making (Scheibe et al., 2010). Furthermore, ACG has been associated with speed-accuracy tradeoff processes that involve a change in the distance between the start and end point of the accumulation process (Forstmann et al., 2008).

For the bias manipulation with potential payoff, we found similar regions of the frontoparietal network that were sensitive to changes in starting point. In addition, we found sensitivity to starting point changes for the left OFC and the right hippocampus. Recently we have shown that OFC and hippocampus play a role in setting starting point values in a similar manipulation, suggesting a specific role for these areas in changing the dynamics of the decision process (Forstmann et al., 2010).

In the scanner session, the biasing effects of the potential payoff on RT and accuracy did not exceed the level of significance. This might be due to a smaller number of trials in the scanner session, resulting in a lower statistical power. However, other studies with similar manipulations report that the biasing effect of a payoff manipulation is less strong than a manipulation of prior probability (Maddox and Bohil, 1998; Simen et al., 2009; Leite and Ratcliff, 2011), suggesting that the diminished effect by the payoff manipulation might have a more systematic cause. One explanation could be that subjects are more sensitive to "being accurate" than "gaining reward," resulting in a more pronounced effect for prior knowledge about the direction of the stimulus, instead of the reward associated with it. This, in turn, might result in a lower activity pattern of brain regions associated with the biasing cue, explaining the smaller effects on BOLD responses for the starting point covariate in the payoff manipulation. Subsequently, although a conjunction analyses is conservative by nature, the smaller effects of payoff on the BOLD signal influences this analyses as well. However, despite the exploratory nature of these analyses, the results are in line with studies of expected value and bias, suggesting that this effect might be more robust than assumed by the lenient threshold (Boettiger et al., 2007; Serences, 2008; Gläscher et al., 2009; Wunderlich et al., 2009; Fleming et al., 2010b; Forstmann et al., 2010; Philiastides et al., 2010; Scheibe et al., 2010; Hare et al., 2011).

We hypothesized that a common mechanism of bias in choice behavior would reflect changes in the BOLD response associated with changes in starting point, regardless of the type of prior information provided by the cue. We identified these regions by performing a conjunction analyses across both manipulations. Five regions show similar effects for both bias manipulations: right medFG, ACG and SFG, left middle temporal gyrus and IPS. Subjects with a higher BOLD signal in these regions had a larger change in starting point for both bias manipulations, suggesting a specific role for these regions in a common mechanism underlying bias in choice behavior. As noted already, these regions have been shown to play a role in decision making as well as in processing prior information. Furthermore, studies using valuebased choice paradigms have suggested a prominent role of coding or computation of values in medFG and ACG (Boettiger et al., 2007; Wunderlich et al., 2009; Fleming et al., 2010b; Philiastides et al., 2010). However, our results suggest that these regions do not necessarily code for value, but rather reflect a common process where a stage is set for a decision that maximizes reward (Feng et al., 2009; Simen et al., 2009; Bogacz et al., 2010).

A question remains where in the brain the representation of prior knowledge is coded, and how this information is processed 
over time in such a way that it causally changes the starting point of the decision process. Results from imaging and modeling studies have shown value-specific effects in early visual areas, suggesting that value might be encoded earlier in the process then has been suggested up until now (Liston and Stone, 2008; Serences, 2008). Furthermore, human and non-human primate research has shown bias effects in regions involved in early processes of saccadic eye movement, suggesting bottom-up processing from the value representation (Lauwereyns et al., 2002; Serences, 2008; Summerfield and Koechlin, 2008; So and Stuphorn, 2010). Although our results are in line with these findings, the low temporal resolution of the BOLD signal does not allow detailed analyses of temporal processes, and as such other methods such as EEG and MEG might help to investigate the causal dynamics of the decision process.

Although our findings of a starting point mechanism are in line with earlier findings, it does not necessarily rule out the possibility that changes in drift rate can also bias choice behavior. It has been suggested that when the difficulty of trials is fixed (as in this study), the starting point rather than drift rate should be adjusted to maximize the rate of receiving reward (Bogacz et al., 2006). However, in case of a variable difficulty across trials, changing drift rate would be optimal. Furthermore, it has been suggested that when the reliability of the sensory evidence differs, bias enters the accumulation process dynamically (Hanks et al., 2011). As such, the optimal strategy of using prior knowledge in decision making might depend on specific conditions such as reliability or uncertainty. In the current study, we fixed the level of difficulty of the motion stimulus at a performance level of $80 \%$ correct. At least under these circumstances, the bias process seems to depend mostly on starting point changes, even for different manipulations of choice bias.

\section{Conclusion}

We used model-based functional MRI to investigate whether bias in choice behavior is similar for different types of prior knowledge. For two different types of prior knowledge we found that changes in the starting point of the evidence accumulation can account for the biasing effects in RT and accuracy of a simple perceptual decision task. Imaging results showed that regions of the frontoparietal network are involved in changing starting points for both manipulations, suggesting a common mechanism underlying the biasing effects of prior knowledge.

\section{References}

Ashby FG (1983) A biased random walk model for two choice reaction times. J Math Psychol 27:277-297.

Basten U, Biele G, Heekeren HR, Fiebach CJ (2010) How the brain integrates costs and benefits during decision making. Proc Natl Acad Sci U S A 107:21767-21772.

Beckmann CF, Jenkinson M, Smith SM (2003) General multilevel linear modeling for group analysis in FMRI. Neuroimage 20:1052-1063.

Boettiger CA, Mitchell JM, Tavares VC, Robertson M, Joslyn G, D'Esposito M, Fields HL (2007) Immediate reward bias in humans: fronto-parietal networks and a role for the catechol-O-methyltransferase 158Val/Val genotype. J Neurosci 27:14383-14391.

Bogacz R (2007) Optimal decision-making theories: linking neurobiology with behaviour. Trends Cogn Sci 11:118-125.

Bogacz R, Brown E, Moehlis J, Holmes P, Cohen JD (2006) The physics of optimal decision making: a formal analysis of models of performance in two-alternative forced-choice tasks. Psychol Rev 113:700-765.

Bogacz R, Hu PT, Holmes P, Cohen JD (2010) Do humans produce the speed-accuracy trade-off that maximizes reward rate? Q J Exp Psychol 63:863-891.

Brainard DH (1997) The psychophysics toolbox. Spat Vis 10:433-436.

Britten KH, Shadlen MN, Newsome WT, Movshon JA (1992) The analysis of visual motion: a comparison of neuronal and psychophysical performance. J Neurosci 12:4745-4765.

Carpenter RH, Williams ML (1995) Neural computation of log likelihood in control of saccadic eye movements. Nature 377:59-62.

Diederich A, Busemeyer JR (2006) Modeling the effects of payoff on response bias in a perceptual discrimination task: Bound-change, drift-rate-change, or two-stage-processing hypothesis. Percept Psychophys 68:194-207.

Edwards W (1965) Optimal strategies for seeking information: Models for statistics, choice reaction times, and human information processing. J Math Psychol 2:312-329.

Feng S, Holmes P, Rorie A, Newsome WT (2009) Can monkeys choose optimally when faced with noisy stimuli and unequal rewards? PLoS Comput Biol 5:e1000284.

Fleming SM, Thomas CL, Dolan RJ (2010a) Overcoming status quo bias in the human brain. Proc Natl Acad Sci U S A 107:6005-6009.

Fleming SM, Whiteley L, Hulme OJ, Sahani M, Dolan RJ (2010b) Effects of category-specific costs on neural systems for perceptual decision-making. J Neurophysiol 103:3238-3247.

Forstmann BU, Dutilh G, Brown S, Neumann J, von Cramon DY, Ridderinkhof KR, Wagenmakers EJ (2008) Striatum and pre-SMA facilitate decision-making under time pressure. Proc Natl Acad Sci USA 105:17538-17542.

Forstmann BU, Brown S, Dutilh G, Neumann J, Wagenmakers E-J (2010) The neural substrate of prior information in perceptual decision making: a model-based analysis. Front Hum Neurosci 4:40.

Forstmann BU, Wagenmakers EJ, Eichele T, Brown S, Serences JT (2011) Reciprocal relations between cognitive neuroscience and formal cognitive models: opposites attract? Trends Cogn Sci 15:272-279.

Gläscher J, Hampton AN, O’Doherty JP (2009) Determining a role for ventromedial prefrontal cortex in encoding action-based value signals during reward-related decision making. Cereb Cortex 19:483-495.

Glimcher PW (2003) The neurobiology of visual-saccadic decision making. Annu Rev Neurosci 26:133-179.

Gold JI, Shadlen MN (2002) Banburismus and the brain: decoding the relationship between sensory stimuli, decisions, and reward. Neuron 36:299-308.

Gold JI, Shadlen MN (2003) The influence of behavioral context on the representation of a perceptual decision in developing oculomotor commands. J Neurosci 23:632-651.

Gold JI, Shadlen MN (2007) The neural basis of decision making. Annu Rev Neurosci 30:535-574.

Hanks TD, Mazurek ME, Kiani R, Hopp E, Shadlen MN (2011) Elapsed decision time affects the weighting of prior probability in a perceptual decision task. J Neurosci 31:6339-6352.

Hansen K, Hillenbrand S, Ungerleider L (2011) Persistency of priorsinduced bias in decision behavior and the fMRI signal. Front Neurosci 5:29.

Hare TA, Schultz W, Camerer CF, O’Doherty JP, Rangel A (2011) Transformation of stimulus value signals into motor commands during simple choice. Proc Natl Acad Sci U S A 108:18120-18125.

Heekeren HR, Marrett S, Bandettini PA, Ungerleider LG (2004) A general mechanism for perceptual decision-making in the human brain. Nature 431:859-862.

Heekeren HR, Marrett S, Ruff DA, Bandettini PA, Ungerleider LG (2006) Involvement of human left dorsolateral prefrontal cortex in perceptual decision making is independent of response modality. Proc Natl Acad Sci U S A 103:10023-10028.

Ho TC, Brown S, Serences JT (2009) Domain general mechanisms of perceptual decision making in human cortex. J Neurosci 29:8675-8687.

Jenkinson M, Smith S (2001) A global optimisation method for robust affine registration of brain images. Med Image Anal 5:143-156.

Jenkinson M, Bannister P, Brady M, Smith S (2002) Improved optimization for the robust and accurate linear registration and motion correction of brain images. Neuroimage 17:825-841.

Kayser AS, Buchsbaum BR, Erickson DT, D'Esposito M (2010) The functional anatomy of a perceptual decision in the human brain. J Neurophysiol 103:1179-1194.

Laming D (1968) Information theory of choice reaction times. New York: Academic.

Lauwereyns J (2010) The anatomy of bias: how neural circuits weigh the options. Cambridge, MA: MIT. 
Lauwereyns J, Watanabe K, Coe B, Hikosaka O (2002) A neural correlate of response bias in monkey caudate nucleus. Nature 418:413-417.

Leite FP, Ratcliff R (2011) What cognitive processes drive response biases? A diffusion model analysis. Judgment and Decision Making 6:651-687.

Link S, Heath R (1975) A sequential theory of psychological discrimination. Psychometrika 40:77-105.

Liston DB, Stone LS (2008) Effects of prior information and reward on oculomotor and perceptual choices. J Neurosci 28:13866-13875.

Maddox WT, Bohil CJ (1998) Base-rate and payoff effects in multidimensional perceptual categorization. J Exp Psychol Learn Mem Cogn 24:1459-1482.

Mulder MJ, Bos D, Weusten JM, van Belle J, van Dijk SC, Simen P, van Engeland H, Durston S (2010) Basic impairments in regulating the speed-accuracy tradeoff predict symptoms of attention-deficit/hyperactivity disorder. Biol Psychiatry 68:1114-1119.

Newsome WT, Paré EB (1988) A selective impairment of motion perception following lesions of the middle temporal visual area (MT). J Neurosci 8:2201-2211.

Nichols T, Brett M, Andersson J, Wager T, Poline JB (2005) Valid conjunction inference with the minimum statistic. Neuroimage 25:653-660.

Oldfield RC (1971) The assessment and analysis of handedness: the Edinburgh inventory. Neuropsychologia 9:97-113.

Palmer J, Huk AC, Shadlen MN (2005) The effect of stimulus strength on the speed and accuracy of a perceptual decision. J Vis 5:376-404.

Pelli DG (1997) The VideoToolbox software for visual psychophysics: transforming numbers into movies. Spat Vis 10:437-442.

Philiastides MG, Sajda P (2007) EEG-informed fMRI reveals spatiotemporal characteristics of perceptual decision making. J Neurosci 27:1308213091.

Philiastides MG, Biele G, Heekeren HR (2010) A mechanistic account of value computation in the human brain. Proc Natl Acad Sci U S A 107:9430-9435.

Preuschhof C, Schubert T, Villringer A, Heekeren HR (2010) Prior information biases stimulus representations during vibrotactile decision making. J Cogn Neurosci 22:875-887.

Ratcliff R (1978) A theory of memory retrieval. Psychol Rev 85:59-108.

Ratcliff R (1985) Theoretical interpretations of the speed and accuracy of positive and negative responses. Psychol Rev 92:212-225.

Ratcliff R, McKoon G (2008) The diffusion decision model: theory and data for two-choice decision tasks. Neural Comput 20:873-922.

Ratcliff R, Tuerlinckx F (2002) Estimating parameters of the diffusion model: approaches to dealing with contaminant reaction times and parameter variability. Psychon Bull Rev 9:438-481.
Ratcliff R, Van Zandt T, McKoon G (1999) Connectionist and diffusion models of reaction time. Psychol Rev 106:261-300.

Scheibe C, Ullsperger M, Sommer W, Heekeren HR (2010) Effects of parametrical and trial-to-trial variation in prior probability processing revealed by simultaneous electroencephalogram/functional magnetic resonance imaging. J Neurosci 30:16709-16717.

Serences JT (2008) Value-based modulations in human visual cortex. Neuron 60:1169-1181.

Simen P, Cohen JD, Holmes P (2006) Rapid decision threshold modulation by reward rate in a neural network. Neural Netw 19:1013-1026.

Simen P, Contreras D, Buck C, Hu P, Holmes P, Cohen JD (2009) Reward rate optimization in two-alternative decision making: empirical tests of theoretical predictions. J Exp Psychol Hum Percept Perform 35:18851897.

So NY, Stuphorn V (2010) Supplementary eye field encodes option and action value for saccades with variable reward. J Neurophysiol 104: 2634-2653.

Summerfield C, Koechlin E (2008) A Neural Representation of Prior Information during Perceptual Inference. Neuron 59:336-347.

Summerfield C, Koechlin E (2010) Economic value biases uncertain perceptual choices in the parietal and prefrontal cortices. Front Hum Neurosci 4:208.

Voss A, Rothermund K, Voss J (2004) Interpreting the parameters of the diffusion model: an empirical validation. Mem Cognit 32:1206-1220.

Wagenmakers EJ (2009) Methodological and empirical developments for the Ratcliff diffusion model of response times and accuracy. Eur J Cogn Psychol 21:641-671.

Wagenmakers EJ, Ratcliff R, Gomez P, McKoon G (2008a) A diffusion model account of criterion shifts in the lexical decision task. J Mem Lang 58:140-159.

Wagenmakers EJ, van der Maas HL, Dolan CV, Grasman RP (2008b) EZ does it! Extensions of the EZ-diffusion model. Psychon Bull Rev 15:1229-1235.

Woolrich MW, Ripley BD, Brady M, Smith SM (2001) Temporal autocorrelation in univariate linear modeling of fMRI Data. Neuroimage 14:1370-1386

Woolrich MW, Behrens TE, Beckmann CF, Jenkinson M, Smith SM (2004) Multilevel linear modelling for FMRI group analysis using Bayesian inference. Neuroimage 21:1732-1747.

Wunderlich K, Rangel A, O'Doherty JP (2009) Neural computations underlying action-based decision making in the human brain. Proc Natl Acad Sci U S A 106:17199-17204. 